\title{
BIBLIOGRAPHY OF SANAMED JOURNAL (2014-2017)
}

\author{
Jašović Ivana, Veselinović Bojana \\ National library of Serbia, Belgrade, Serbia
}

Primljen / Received 12. 01. 2018. god.

Abstract: This paper presents a bibliography of Sanamed, journal of medical and health science, founded by a group of doctors from Novi Pazar. The Journal published original articles, case reports, literature reviews, articles on history of medicine, articles for practitioners, book reviews, and other medical information in the field of medicine, dentistry, pharmacology and pharmaceutical sciences. The bibliography contains 101 bibliographic units and includes the period from 2014 to 2017 (4 years). The aim of this bibliography is to be exhanstive at including all the contribution published in all the numbers of individual years. The material is completely formally described and all bibliography units are objectively classified - they have UDK numbers and subjects, which are systematized in the subject sense

\section{INTRODUCTION}

Sanamed is journal of medical and health science, founded 2006 by a group of doctors from Novi Pazar. The Journal published original articles, case reports, literature reviews, articles on history of medicine, articles for practitioners, book reviews, and other medical information in the field of medicine, dentistry, pharmacology and pharmaceutical sciences. This is an open access journal. From 2006 to 2013, the Journal was published twice a year, since 2014, it was published three times a year.

Editor-in-Chief is prim. Dr. Avdo Ćeranić and assosiate editors are dr. Džemail Detanac and dr. Dženana Detanac.

The Journal is published both in electronic and print format.

This bibliography of periodicals is in its character special bibliography and it was made up „de visu“.

The bibliography contains 101 bibliographic units and includes the period from 2014 to 2017 (4 years). The aim of this bibliography is to be exhanstive at including all the contribution published in all the num-
Prihvaćen/Accepted 20. 02. 2018. god.

bers of individual years. It should be noted that such contribution as introduction parts, editorials, book reviews, advertisements, etc. they were no processed due to their negligible informative value and importance for the journal.

Since the elements for the bibliographic description are taken from primary source, the cover pages and preliminaries of journal, the bibliography has the primary character. During the bibliographic work, a letter and a language were respected and subject heading is cyrillic.

The material is completely formally described and all bibliography units are objectively classified - they have UDK numbers and subjects, which are systematized in the subject sense.

The bibliography was made in accordance with the International Standard ISBD (CP), communicated format for machine-readable cataloging and exchange bibliographic informations - COMARC/B, and in accordance with "The Rulebook and the Manual for the Preparation Alphabetical Catalogs I and II" by Eva Verona, and other expert literatures (professional dictionaries, encyclopedias, lexicons, electronic databases etc.).

Bibliographic material is sorted according to the alphabetical order of the surname authors of articles, contributions, or titles of articles if they are treated as anonymous work in catalog sense.

\section{Bibliography of Sanamed Journal (2014-2017)}

3 Social sciences

321 Forms of political organization. States as political powers

SM - 1

BLIND Serbian Rulers and Famous Persons / Janicijevic Katarina ... [et al.]. - Ill. - Available on: http: //scindeks-clanci.ceon.rs/daa/pdf/1452-662X/2016/1452662X1603249J.pdf. - Available on: http://www.sana- 
med.rs/OJS/index.php/Sanamed/issue/view/6. - Other authors: Sanja Kocić, Snežana Radovanović, Tatjana Šarenac Vulović, Nenad Petrović, Dušan Todorović. Notes and bibliographical references with the text. Abstract; Sažetak. - Bibliography: p. 324-327.

IN: Sanamed. - ISSN 1452-662X. - Vol. 11, no. 3 (2016), 305-328.

doi: 10.5937/sanamed1603249J

321.17:929 Стефан Дечански, српски краљ; 321.17:929 Стефан Бранковић; 821.163.41:929 Вишњић $\Phi$.

\section{Applied sciences. Medicine. Technology 60 General questions of the applied sciences}

SM - 2

REGULATORY Considerations of Biosimilars and Clinical Dilema of their Use / Stavrik Ganadieva Sonja ... [et al.]. - Ill. - Available on: http://www.sanamed.rs/OJS/index.php/Sanamed/article/view/158/85.

- Notes and bibliographical references with the text. Abstract; Sažetak. - Bibliography: p. 49-50.

IN: Sanamed. - ISSN 1452-662X. - Vol. 12, no. 1 (2017), 45-50.

doi: $10.24125 /$ sanamed.v1i1.158

606:615.3

612 Physiology. Human and comparative physiology

SM - 3

PEZELJ-Ribarić, Sonja, 1966-

Saliva as a Diagnostic fluid / Pezelj-Ribaric Sonja, PrpicJelena, Glazar Irena. - Available on: http:// scindeks-clanci.ceon.rs/data/pdf/1452-662X/2015/1452662X1503215P.pdf. - Available on: http://www.sanamed.rs/OJS/index.php/Sanamed/issue/view/5. - Abstract; Sažetak. - Bibliography: p. 218-219.

IN: Sanamed. - ISSN 1452-662X. - Vol. 10, no. 3 (2015), 215-219.

doi: $10.5937 /$ sanamed $1503215 \mathrm{P}$

612.313.015; 616-074

613 Hygiene generally

SM - 4

\section{ABDELBASSET, Walid Kamal Mohammed, 1979-}

Relationship Between Physical Activity and Health-Related Quality of Life in Elderly People : Across-Section Study / Kamal M. AbdelbassetWallid, Nambi S. Gopal. - Available on: http://www.sanamed.rs/OJS/index.php/Sanamed/article/view/186/99.

- Notes and bibliographical references with the text. Abstract; Sažetak. - Bibliography: p. 91-92.
IN: Sanamed. - ISSN 1452-662X. - Vol. 12, no. 2 (2017), 87-92.

doi: 10.24125/sanamed.v12i2.186

613.72/.73-053.9; 613.98

SM - 5

GOPAL, Nambi S., 1978-

Additional Effect of Trigger Point Therapy and Myofascial Release on Second Stage Frozen Shoulder Among Industrial Workers / Gopal Nambi S., Wallid Kamal M. Abdelbasset. - Tables, ill. - Available on: http://www.sanamed.rs/OJS/index.php/Sanamed/ article/view/187/100. - Notes and bibliographical references with the text. - Abstract; Sažetak. - Bibliography: p. 99-100.

IN: Sanamed. - ISSN 1452-662X. - Vol. 12, no. 2 (2017), 93-100.

doi: $10.24125 /$ sanamed.v12i2.187

613.65:66/67-057; 616.727.2-085.82

SM - 6

MALNUTRITION in the Surgical Patients / Andonovska J. Biljana ... [et al.]. - Ill. - Available on: http://scindeks-clanci.ceon.rs/data/pdf/1452-662X/2016/ 1452-662X1603229A.pdf. - Available on: http://www. sanamed.rs/OJS/index.php/Sanamed/issue/view/7. - Notes and bibliographical references with the text. - Abstract; Sažetak. - Bibliography: p. 236-237.

IN: Sanamed. - ISSN 1452-662X. - Vol. 11, no. 3 (2016), 229-237.

doi: $10.5937 /$ sanamed1603229A

$613.24 / .25-056.24$

SM - 7

WHEN to Postpone Cataract Surgery: Taking in Consideration Patients' Quality of Life / Jovanovic Milos ... [et al.]. - Tables. - Available on: http://scindeks-clanci.ceon.rs/data/pdf/1452-662X/2015/1452662X1503179J.pdf. - Available on: http://www.sanamed.rs/OJS/index.php/Sanamed/issue/view/5. - Other authors: Glisic Selimir, Stanković Zora, Dačić Krnjaja Bojana. - Abstract; Sažetak. - Bibliography: p. 184.

IN: Sanamed. - ISSN 1452-662X. - Vol. 10, no. 3 (2015), 179-184.

doi: $10.5937 /$ sanamed1503179J

613-056.24:617.741-004.1

COBISS.SR-ID 219458828

614 Public health and hygiene.

Accident prevention

SM - 8

The CHARACTERISTICS of the Health State Population in Central Serbia / Radovanovic Snezana ... [et al.]. - Tables. - Available on: http://scindeks-clan- 
ci.ceon.rs/data/pdf/1452-662X/2016/1452-662X1603211R. pdf. - Available on: http://www.sanamed.rs/OJS/index.php/Sanamed/issue/view/7. - Other authors: Sanja Kocić, Dragan Vasiljević, Svetlana Radević, Katarina Janićijević, Nataša Mihailović. - Notes and bibliographical references with the text. - Abstract; Sažetak. - Bibliography: p. 216.

IN: Sanamed. - ISSN 1452-662X. - Vol. 11, no. 3 (2016), 211-216.

doi: $10.5937 /$ sanamed1603211R

614.2(497.11)"2015"

615 Pharmacology. Therapeutics. Toxicology

SM - 9

ANALYZES of Antiplatelets and Anticoagulants Utilization in Patients Treated in Cardiovascular RehavilitionCentar from Croatia / Boban Marko ... [et al.]. - Tables. - Available on: http://scindeks-clanci.ceon.rs/data/pdf/1452-662X/2016/1452662X1602099B.pdf. - Available on: http:/www.sanamed.rs/OJS/index.php/Sanamed/issue/view/7. - Other authors: Persić Viktor, Pesa Vladimir, Pehar-Pejcinović Vesna, Detanac S. Dzemail, Sipić Tomislav, Zulj Marinko, Franović Tamara, Vcev Aleksandar. - Notes and bibliographical references with the text. - Abstract; Sažetak. - Bibliography: p. 106-107.

IN: Sanamed. - ISSN 1452-662X. - Vol. 11, no. 2 (2016), 99-107.

doi: $10.5937 /$ sanamed1602099B

615.273 .3

\section{SM - 10}

GLAŽAR, Irena, 1973-

The Effect of Fluconazole and Amphotericin B on Macrophage Functions / Glazar Irena, Pezelj-Ribaric Sonja, Abram Maja. - Tables. - Available on: http: //scindeks-clanci.ceon.rs/data/pdf/1452-662X/2015/ 1452-662X1503173G.pdf. - Available on: http://www. sanamed.rs/OJS/index.php/Sanamed/issue/view/5. - Abstract; Sažetak. - Bibliography: p. 176-177.

IN: Sanamed. - ISSN 1452-662X. - Vol. 10, no. 3 (2015), 173-177.

doi: $10.5937 /$ sanamed $1503173 \mathrm{G}$

615.281 .076

SM - 11

\section{DUSPER, Silva, 1965-}

Vasoactive Drugs in Critical Care / Dusper Silva, HusedžinovićIno. - Tables. - Available on: http://scindeks-clanci.ceon.rs/data/pdf/1452-662X/2014/ 1452-662X 1402185D.pdf. - Available on: http://sanamed. rs/sanamed_pdf/sanamed_9_2/Dusper_Silva.pdf. - Abstract; Sažetak. - Bibliography: p. 189-190.
IN: Sanamed. - ISSN 1452-662X. - Vol. 9, no. 2 (2014), 185-190.

$615.22 .031 / .038$

SM - 12

\section{ŽIVKOVIĆ, Jovan}

The Effects of Chronic Lead Poisoning on the Values of Hypertension in Children / Zivkovic Jovan, Savic Zoran. - Ill.. - Available on: http://scindeks-clanci.ceon.rs/data/pdf/1452-662X/2014/1452662X1403215Z.pdf. - Available on: http://sanamed. rs/sanamed_pdf/sanamed_9_3/Zivkovic_Jovan.pdf. Abstract; Sažetak. - Bibliography: p. 221-222.

IN: Sanamed. - ISSN 1452-662X. - Vol. 9, no. 3 (2014), 215-222.

615.9:546.815(497.115);

616.12-008.331.1-053.2(497.115)

616 Pathology. Clinical medicine

SM - 13

ACUTE Renal Failure in Term Newborn Following Perinatal Asphyxia / HadzimuratovicEmina ... [et al.]. - Ill., tables. - Available on: http://www.sanamed.rs/OJS/index.php/Sanamed/article/view/162. - Notes and bibliographical references with the text. - Abstract; Sažetak. - Bibliography: p. 14

IN: Sanamed. - ISSN 1452-662X. - Vol. 12, no. 1 (2017), 11-14.

doi: 10.24125/sanamed.v1i1.162

616-001.8-053.31; 616.61-008.6-053.31

SM - 14

BENEFICIAL Effects of Levothyroxine in the Treatment of Subclinical Hypothyroidism / Mulić Mersudin ... [et al.]. - Ill. - Available on: http://scindeks-clanci.ceon.rs/data/pdf/1452-662X/2016/1452662X1603203M.pdf. - Available on: http://www.sanamed.rs/OJS/index.php/Sanamed/issue/view/7. - Other authors: Halo Orhan, Škrijelj Fadil, Mulić Bilsana. Notes and bibliographical references with the text. Abstract; Sažetak. - Bibliography: p. 208-209.

IN: Sanamed. - ISSN 1452-662X. - Vol. 11, no. 3 (2016), 203-209.

doi: $10.5937 /$ sanamed $1603203 \mathrm{M}$

616.441-008.64-085.357; 615.357:577.175.4

SM - 15

BRUGADA Syndrome - A Case Report / Kuzevska-ManevaKonstandina ... [et al.]. - Ill. - Available on: http://scindeks-clanci.ceon.rs/data/pdf/1452-662X/ 2016/1452-662X1601053K.pdf. - Available on: http://www.sanamed.rs/OJS/index.php/Sanamed/issue/ view/6. - Abstract; Sažetak. - Bibliography: p. 56. 
IN: Sanamed. - ISSN 1452-662X. - Vol. 11, no. 1 (2016), 53-56.

doi: $10.5937 /$ sanamed $1601053 \mathrm{~K}$

616.12-008.313

\section{SM - 16}

VALIDITY of Core Needle Biopsy in the Histopathological Verification of Parotid Gland Lesions / Oroz Aleksandar ... [et al.]. - Ill. - Available on: http: //scindeks-clanci.ceon.rs/data/pdf/1452-662X/2016/ 1452-662X1602123O.pdf. - Available on: http://www. sanamed.rs/OJS/index.php/Sanamed/issue/view/7. - Other authors: Kanjevac Tatjana, Vasović Miroslav, Milošević Marija, Jevdjić Jasna. - Notes and bibliographical references with the text. - Abstract; Sažetak. - Bibliography: p. 126-127.

IN: Sanamed. - ISSN 1452-662X. - Vol. 11, no. 2 (2016), 123-127.

doi: $10.5937 /$ sanamed 16021230

616.447-076

SM - 17

VOLKMANN'S contracture as a Complication of Supracodylar Fracture of Humerus in Children Case Report / Bozinovski Zoran ... [et al.]. - Ill. - Available on: http://scindeks-clanci.ceon.rs/data/pdf/1452662X/2016/1452-662X1601057B.pdf. - Available on: http://www.sanamed.rs/OJS/index.php/Sanamed/issue/ view/6. - Abstract; Sažetak. - Bibliography: p. 61.

IN: Sanamed. - ISSN 1452-662X. - Vol. 11, no. 1 (2016), 57-61.

doi: $10.5937 /$ sanamed1601057B

616.717.4-001.5-06-053.2

SM - 18

GENERAL Anesthesia : Is it Safe for Newborns, Infants and Young Children? / Nancheva Jasminka ... [et al.]. - Available on: http://scindeks-clanci.ceon.rs/ data/pdf/1452-662X/2016/1452-662X1602163N. pdf. Available on: http://www.sanamed.rs/OJS/index.php/ Sanamed/issue/view/7. - Other authors: Kamnar Viktor, Nenceva Andrea, Georgieva Daniela, Georgiev Antonio, Andonovski Alan. - Notes and bibliographical references with the text. - Abstract; Sažetak. - Bibliography: p. 167.

IN: Sanamed. - ISSN 1452-662X. - Vol. 11, no. 2 (2016), 163-167.

doi: $10.5937 /$ sanamed $1602163 \mathrm{~N}$

616-089.5-053.2

SM - 19

GEORGIEV, Antonio, 1969-

Cocaine Cardiomyopathy - A Case Report / Georgiev Antonio, Zhivadinovik Julija. - Ill. - Available on: http://www.sanamed.rs/OJS/index.php/Sanamed/article/view/166. - This article is a correction of the article published In Sanamed Medical Journal, vol. 9, issue 3, in the text titled "Cocaine cardiomiopathi-A case report". Authors made a mistake by inadequately citing parts of scientific paper, in sections Introduction and Discussion. - Abstract; Sažetak. - Bibliography: p. 259.

IN: Sanamed. - ISSN 1452-662X. - Vol. 11, no. 3 (2016), 255-259.

616.127-056.83(497.7); 613.83(497.7)

SM - 20

\section{GEORGIEV, Antonio, 1969-}

Cocaine Cardiomyophathy - A case Report / Georgiev Antonio, Zhivadinovik Julija. - Ill. - Available on: http://scindeks-clanci.ceon.rs/data/pdf/1452-662X/ 2014/1452-662X1403233G.pdf. - Available on: http:// sanamed.rs/sanamed_pdf/sanamed_9_3/Georgiev_Anto nio.pdf. - The correction of this article was published InSanamed Medical Journal, vol. 11, issue 3, in the text titled "Cocaine cardiomiopathi-A case report". Authors made a mistake by inadequately citing parts of scientific paper, in sections Introduction and Discussion. - Abstract; Sažetak. - Bibliography: p. 236-237.

IN: Sanamed. - ISSN 1452-662X. - Vol. 9, no. 3 (2014), 233-237.

616.127-056.83(497.7); 613.83:616.127

SM - 21

GRUJIĆ-Vujmilović, Dragana, 1975-

Quality of Life of Patients with Diabetes Mellitus - Social Domain of Health / Grujić-Vujmilović Dragana, Gavrić Živan. - Tables. - Available on: http://scindeks-clanci.ceon.rs/data/pdf/1452-662X/2014/ 1452-662X1402151G.pdf. - Available on: http://sanamed.rs/sanamed_pdf/sanamed_9_2/Grujic_Vujmilovic_Dragana.pdf. - Abstract; Sažetak. - Bibliography: p. 158-159.

IN: Sanamed. - ISSN 1452-662X. - Vol. 9, no. 2 (2014), 151-159.

613-056.24:616.379-008.64

SM - 22

DOES the Addition of Dexsamethason to Local Anesthetic Prolong the Analgesia of Interscalen Plexus Brachialis Block in Patients with Shoulder Surgery? / Nanceva Jasminka ... [et al.]. - Tables. Available on: http://scindeks-clanci.ceon.rs/data/pdf/ 1452-662X/2016/1452-662X1601015N.pdf. - Available on: http://www.sanamed.rs/OJS/index.php/Sanamed/issue/view/6. - Abstract; Sažetak. - Bibliography: p. 20. 
IN: Sanamed. - ISSN 1452-662X. - Vol. 11, no. 1 (2016), 15-20.

doi: $10.5937 /$ sanamed $1601015 \mathrm{~N}$

616.853-001-089.5

SM - 23

EARLY signs of diabetic nephrophathy and ultrasound characteristics of kidneysin children and youth with Diabetes mellitus type 1 / EvlijanaHasanovic ... [et al.]. - Ill. - Available on: http://scindeks-clanci.ceon.rs/data/pdf/1452-662X/2014/1452662X1401041H.pdf. - Available on: http://sanamed.rs/ sanamed_pdf/sanamed_9_1/Evlijana_Hasanovic.pdf. - Other authors: Fahrija Skokic, Belkisa Colic, Almira Cosickic, Midhat Hajder, Goran Imamovic, Senaid Trnacevic. - Abstract; Sažetak. - Bibliography: p. 48.

IN: Sanamed. - ISSN 1452-662X. - Vol. 9, no. 1 (2014), 41-48.

616.61:616.379-008.64

SM - 24

EVALUATION of Two Surgical Treatments of Primary Vesicoureteral Reflux Among Children : A 15 Years Experience / Memeti Shaban ... [et al.]. - Ill.. - Available on: http://scindeks-clanci.ceon.rs/data/pdf/1452-662X/2016/1452-662X1602093M.pdf. Available on: http://www.sanamed.rs/OJS/index.php/ Sanamed/issue/view/7. - Other authors: Petrovski Mile, Petrovski Pero, Simenov Risto, Kamilovski Marian, Todorović Lazar, Ziberi Jetmir, Ristevski Toni, Lumani Njomza, Mihajlović Mila, Ristovska Gordana. - Notes and bibliographical references with the text. - Abstract; Sažetak. - Bibliography: p. 97.

IN: Sanamed. - ISSN 1452-662X. - Vol. 11, no. 2 (2016), 93-97.

doi: $10.5937 /$ sanamed $1602093 \mathrm{M}$

616.61-007-089-053.2(497.7)"1999/2004"

\section{SM - 25}

The EFFECT of Plasma Preparation Rich in Growth Factors on Patellar Stability After Medial Patellofemoral Ligament Reefing / Andonovski Alan ... [et al.]. - Ill. - Available on: http://scindeks-clanci. ceon.rs/data/pdf/1452-662X/2016/1452-662X1602109A. pdf. - Available on: http://www.sanamed.rs/OJS/index.php/Sanamed/issue/view/7. - Other authors: Nencheva Jasminka, Andonovska Biljana, Petrovska-Cvetkovska Dragana, Nenceva Andrea, Alabakovska Sonja. - Notes and bibliographical references with the text. - Abstract; Sažetak. - Bibliography: p. 114-115.

IN: Sanamed. - ISSN 1452-662X. - Vol. 11, no. 2 (2016), 109-115. doi: $10.5937 /$ sanamed1602109A

616.728.3-089

SM - 26

ECTOPIC Pancreatic Tissue in the Stomach : Case Report / LukićDejan ... [et al.]. - Ill. - Available on: http://www.sanamed.rs/OJS/index.php/Sanamed/ article/view/171/89. - Other authors: Tatić Milanka, Radovanović Zoran, Ranisavljević Milan, Kresoja Milana, Đurić Mladen. - Notes and bibliographical references with the text. - Abstract; Sažetak. - Bibliography: p. 36.

IN: Sanamed. - ISSN 1452-662X. - Vol. 12, no. 1 (2017), 33-36.

doi: $10.24125 /$ sanamed.v1i1.171

$616.37-007.41$

SM - 27

\section{ZDRAVKOVIĆ, Ivana}

Safe extubation and reintubation in operatory room and ICU patients / Ivana Zdravković, Radmilo J. Janković. - Available on: http://scindeks-clanci. ceon.rs/data/pdf/1452-662X/2014/1452-662X1401089Z. pdf. - Available on: http://sanamed.rs/sanamed_pdf/sanamed_9_1/Ivana_Zdravkovic.pdf. - Abstract; Sažetak. - Bibliography: p. 96-98.

IN: Sanamed. - ISSN 1452-662X. - Vol. 9, no. 1 (2014), 89-98.

616-089.819.3

SM - 28

\section{ZENEV, Ivan}

Arytenoidcordectomy for Bilateral Vocal Cord Paralysis: Primary and Revision Procedure / Zenev Ivan, Sapundzhiev Nikolay. - Ill. - Available on: http://scindeks-clanci.ceon.rs/data/pdf/1452-662X/2015/ 1452-662X1501023Z.pdf. - Available on: http://www. sanamed.rs/OJS/index.php/Sanamed/issue/view/2. - Abstract; Sažetak. - Bibliography: p. 29.

IN: Sanamed. - ISSN 1452-662X. - Vol. 10, no. 1 (2015), 23-29.

doi: $10.5937 /$ sanamed $1501023 \mathrm{Z}$

616.225-009.1-089

SM - 29

\section{ZULIĆ, Evlijana}

Acute Renal Failure in the Newborns Hospitalized at the Intensive Care Unit, University Clinica Centre Tuzla / Zulic Evlijana, Hadzic Devleta. - Tables. - Available on: http://scindeks-clanci.ceon.rs/data/pdf/1452-662X/2015/1452-662X1501047Z.pdf. Available on: http://www.sanamed.rs/OJS/index.php/ 
Sanamed/issue/view/2. - Abstract; Sažetak. - Bibliography: p. 50.

IN: Sanamed. - ISSN 1452-662X. - Vol. 10, no. 1 (2015), 47-50.

doi: $10.5937 /$ sanamed $1501047 \mathrm{Z}$

616.61-008.64-053.31(497.6)

SM - 30

INTRACAPSULAR and Para-articular Chondroma of Knee: Case Report / Temelkovski Zlatko ... [et al.]. - Ill. - Available on: http://www.sanamed. rs/OJS/index.php/Sanamed/article/view/170. - Notes and bibliographical references with the text. - Abstract; Sažetak. - Bibliography: p. 30-31.

IN: Sanamed. - ISSN 1452-662X. - Vol. 12, no. 1 (2017), 27-31.

doi: 10.24125/sanamed.v1i1.170

616.728.3-006.33

SM - 31

INFRAPATELLAR Fat Pad Haemangioma Case Report / Dzoleva-Tolevska Roza ... [et al.]. Photogr. - Available on: http://scindeks-clanci.ceon.rs/ data/pdf/1452-662X/2014/1452-662X1402177D.pdf. - Available on: http://sanamed.rs/sanamed pdf/sanamed 9 2/Dzoleva_Tolevska_Roza.pdf. - Other authors: Poposka Anastasika, Samardziski Milan, Georgieva Daniela. - Abstract; Sažetak. - Bibliography: p. 180.

IN: Sanamed. - ISSN 1452-662X. - Vol. 9, no. 2 (2014), 177-180.

$616.728 .3-006.3$

SM - 32

\section{JOWITT, Ljiljana, 1953-}

Ethnicity and Type 2 Diabetes in Asian Indian Migrants in Auckland, New Zealand / Jowitt Ljiljana. - Tables. - Available on: http://scindeks-clanci.ceon. rs/data/pdf/1452-662X/2014/1452-662X1403253J.pdf. - Available on: http://sanamed.rs/sanamed_pdf/sanamed_9_3/Jowitt_Ljiljana.pdf. - Abstract; Sažetak. Bibliography: p. 261-263.

IN: Sanamed. - ISSN 1452-662X. - Vol. 9, no. 3 (2014), 253-263.

616.379-008.64-054.7(931)

SM - 33

\section{KAKKAR, Apeksha}

The Subtypes of Pancreatic Ductal Adenocarcinomas / Kakkar Apeksha, Choudhuri Jui, Mukherjee Indraneil. - Ill. - Available on: http://scindeks-clanci. ceon.rs/data/pdf/1452-662X/2016/1452-662X1603239A. pdf. - Available on: http://www.sanamed.rs/OJS/index.php/Sanamed/issue/view/7. - Abstract; Sažetak. Bibliography: p. 241-242.
IN: Sanamed. - ISSN 1452-662X. - Vol. 11, no. 3 (2016), 239-242.

doi: $10.5937 /$ sanamed $1603239 \mathrm{~K}$

616.37-006.6

SM - 34

\section{KAMNAR, Viktor}

Total Knee Surgery : Our Experience / Kamnar Viktor, Poposka Anastasika, Doksevska Bogojevska Milena. - Photogr., tabela. - Notes and bibliographical references with the text. - Abstract; Sažetak. - Bibliography: p. 107-108.

IN: Sanamed. - ISSN 1452-662X. - Vol. 12, no. 2 (2017), 101-108.

doi: 10.24125/sanamed.v12i2.190

616.728.3-089.844

SM - 35

\section{KOSTOV, Hristijan}

Comparison of clinical and arthroscopic findings in meniscal tears / Kostov Hristijan, Kostova Elena. - Available on: http://scindeks-clanci.ceon.rs/ data/pdf/1452-662X/2014/1452-662X1401025K.pdf. - Available on: http://sanamed.rs/sanamed_pdf/sanamed_9_1/Kostov_Hristijan.pdf. - Abstract; Sažetak. Bibliography: p. 30.

IN: Sanamed. - ISSN 1452-662X. - Vol. 9, no. 1 (2014), 25-30.

616.728.3-001

SM - 36

\section{LEVENTOGIANNIS, Konstantinos}

Intravenous clarithromycin : a valuable immunomodulator for severe infections / Konstantinos Leventogiannis, Evangelos J. Giamarellos-Bourboulis. Available on: http://scindeks-clanci.ceon.rs/data/pdf/ 1452-662X/2014/1452-662X1401083L.pdf. - Available on: http://sanamed.rs/sanamed pdf/sanamed 9 1/ Konstantinos_Leventogian.pdf. - Abstract; Sažetak. Bibliography: p. 87.

IN: Sanamed. - ISSN 1452-662X. - Vol. 9, no. 1 (2014), 83-87.

616-002-085.33.035; 615.33.035

SM - 37

\section{MARKOVIĆ, Ivan, 1966-}

Sentinel Lymph Node Concept in Differentiated Thyroid Cancer / Markovic Ivan, Dzodic Radan. Ill.. - Available on: http://sanamed.rs/sanamed pdf/sanamed 9 3/Markovic Ivan.pdf. - Available on: http:// scindeks-clanci.ceon.rs/data/pdf/1452-662X/2014/1452662X1403239M.pdf. - Abstract; Sažetak. - Bibliography: p. 244-245. 
IN: Sanamed. - ISSN 1452-662X. - Vol. 9, no. 3 (2014), 239-245.

616.441-006.6-033.2; 616.428-033.2

SM - 38

MILISAVLEVIĆ, Slobodan, 1965-

Pneumothorax - Diagnosis and Treatment / Milisavljevic Slobodan, Spasic Marko, Milosevic Bojan. - Tables, ill. - Available on: http://scindeks-clanci. ceon.rs/data/pdf/1452-662X/2015/1452-662X1503221M. pdf. - Available on: http://www.sanamed.rs/OJS/index.php/Sanamed/issue/view/5. - Abstract; Sažetak. Bibliography: p. 228.

IN: Sanamed. - ISSN 1452-662X. - Vol. 10, no. 3 (2015), 221-228.

doi: $10.5937 /$ sanamed1503221M

616.25-003.219

SM - 39

\section{MICEKOWIEC, Dawid}

MicroRNAs as Biomarkers for Acute Myocardial Infarction-Small Molecules with a Huge Potential / Miœkowiec Dawid, Kasprzak Jaroslaw D. - Available on: http://scindeks-clanci.ceon.rs/data/pdf/1452662X/2015/1452-662X1502127M.pdf. - Available on: http://www.sanamed.rs/OJS/index.php/Sanamed/article/view/32. - Abstract; Sažetak. - Bibliography: p. 133-135.

IN: Sanamed. - ISSN 1452-662X. - Vol. 10, no. 2 (2015), 127-135.

doi: $10.5937 /$ sanamed $1502127 \mathrm{M}$

616.127-005.8-074:577.113

SM - 40

\section{MUHVIĆ-Urek, Miranda, 1972-}

Oral Manifestations of Crohn's: A Case Report / Muhvic Urek Miranda, Mijandrusic Sincic Brankica, Braut Alen. - Available on: http://scindeks-clanci.ceon. rs/data/pdf/1452-662X/2015/1452-662X1503205M.pdf. - Available on: http://www.sanamed.rs/OJS/index. php/Sanamed/issue/view/5. - Abstract; Sažetak. - Bibliography: p. 207-208.

IN: Sanamed. - ISSN 1452-662X. - Vol. 10, no. 3 (2015), 205-208.

doi: $10.5937 /$ sanamed $1503205 \mathrm{M}$

616.34-002-06; 616.31

\section{SM - 41}

PERINATAL Outcome of Preterm Infants in Federation of Bosnia and Herzegovina / Skokic Fehrija ... [et al.]. - Ill.. - Available on: http://scindeksclanci.ceon.rs/data/pdf/1452-662X/2015/1452-662X
1501015S.pdf. - Available on: http://www.sanamed.rs/OJS/index.php/Sanamed/issue/view/2 . - Abstract; Sažetak. - Bibliography: p. 22.

IN: Sanamed. - ISSN 1452-662X. - Vol. 10, no. 1 (2015), 15-22.

doi: $10.5937 /$ sanamed $1501015 \mathrm{~S}$

616-053.31(497.6)

\section{SM - 42}

PILOT Study of Contact Sensitization of Formaldehyde-Releasers, Formaldehyde and Glutaraldehyde in Dental Students / Lyapina Maya ... [et al.]. - Tables. - Available on: http://scindeks-clanci.ceon. rs/data/pdf/1452-662X/2016/1452-662X1601021L.pdf. - Available on: http://www.sanamed.rs/OJS/index. php/Sanamed/issue/view/6. - Abstract; Sažetak. - Bibliography: p. 26-27.

IN: Sanamed. - ISSN 1452-662X. - Vol. 11, no. 1 (2016), 21-27.

doi: $10.5937 /$ sanamed $1601021 \mathrm{~L}$

616.248-057.875

SM - 43

PORTAL Vein Thrombosis : Ultrasound Imaging / Trajkovska Meri ... [et al.]. - Ill. - Available on: http://scindeks-clanci.ceon.rs/data/pdf/1452-662X/2016/ 1452-662X1602157T.pdf. - Available on: http://www. sanamed.rs/OJS/index.php/Sanamed/issue/view/7. - Other authors: Popova-Jovanovska Rozalinda, Avramovski Vladimir, Joksimovik Nenad. - Notes and bibliographical references with the text. - Abstract; Sažetak. - Bibliography: p. 161-162.

IN: Sanamed. - ISSN 1452-662X. - Vol. 11, no. 2 (2016), 157-162.

doi: $10.5937 /$ sanamed $1602157 \mathrm{~T}$

616.14-005.6-073

SM - 44

PREDICTORS of Carotid Intima media Thickness in Obese Adolescents / Paripovic Dusan ... [et al.]. - Tables. - Available on: http://www.sanamed. rs/OJS/index.php/Sanamed/article/view/174. - Other authors: Vukomanović Goran, Čivčić Milorad, Peco-Antić Amira. - Notes and bibliographical references with the text. - Abstract; Sažetak. - Bibliography: p. 18-19.

IN: Sanamed. - ISSN 1452-662X. - Vol. 12, no. 1 (2017), 15-19.

doi: $10.24125 /$ sanamed.v1i1.174

616.1-02-053.6; 613.25-053.6

SM - 45

PROGNOSTIC Significance of Serum NGAL Against AKI in ICU Elder Patients after Open Hea- 
rt Surgery / Mosa F. Osama ... [et al.]. - Tables, ill. Notes and bibliographical references with the text. Abstract; Sažetak. - Bibliography: p.

IN: Sanamed. - ISSN 1452-662X. - Vol. 12, no. 2 (2017),

616.1/.2-089-06; 616.61-074

SM - 46

PROTECTIVE mechanical ventilation in patients without or with lung injury / Yuda Sutherasan ... [et al.]. - Ill.. - Available on: http://scindeks-clanci. ceon.rs/data/pdf/1452-662X/2014/1452-662X1401071S. pdf. - Available on: http://sanamed.rs/sanamed_pdf/sanamed_9_1/Yuda_Sutherasan.pdf. - Other authors: Maria Vargas, Raquel Rodríguez-González, Paolo Pelosi. - Abstract; Sažetak. - Bibliography: p. 79-82.

IN: Sanamed. - ISSN 1452-662X. - Vol. 9, no. 1 (2014), 71-82.

$616.24-084 ; 615.816 .2$

SM - 47

RADIAL Artery Anomalies in the Macedonian Population During Transradial Angiogrphy Procedures / Zafirovska Biljana ... [et al.]. - Ill.. - Available on: http://scindeks-clanci.ceon.rs/data/pdf/1452-662X/ 2016/1452-662X1602087Z.pdf. - Available on: http:// www.sanamed.rs/OJS/index.php/Sanamed/issue/view/7. - Other authors: Petkoska Danica, Antov Slobodan, Vasilev Ivan, Jovkovski Aleksandar, Kalpak Oliver, Kostov Jorgo, Spiroski Igor, Pejkov Hristo, Bosev Marjan, Tatavari Hajber, Kitanovski Darko, Kedev Saško. - Notes and bibliographical references with the text. - Abstract; Sažetak. - Bibliography: p. 91-92.

IN: Sanamed. - ISSN 1452-662X. - Vol. 11, no. 2 (2016), 87-92.

doi: $10.5937 /$ sanamed $1602087 \mathrm{Z}$

616.131-007.4(497.7)

SM - 48

The ROLE of Regional Centers and University Children's Hospital in Development of Home Mechanical Ventilation Network / Rsovac Snežana ... [et al.]. - Tables. - Available on: http://www.sanamed.rs /OJS/index.php/Sanamed/article/view/192/103. - Other authors: Milan Đukić, Jasna Kalanj, Biljana Međo, Marko Petrović. - Notes and bibliographical references with the text. - Abstract; Sažetak. - Bibliography: p. 123.

IN: Sanamed. - ISSN 1452-662X. - Vol. 12, no. 2 (2017), 121-123.

doi: $10.24125 /$ sanamed.v12i2.192

616.2-036.1-053; 615.816.2

SM - 49

SEVERE Community-Acquired Pneumonia Caused by Mycoplasma Pneumoniae in Young Fe- male Patient / MilacicNena ... [et al.]. - Ill., tables. Available on: http://scindeks-clanci.ceon.rs/data/pdf/ 1452-662X/2015/1452-662X1502119M.pdf. - Available on: http://www.sanamed.rs/OJS/index.php/Sanamed/article/view/32. - Abstract; Sažetak. - Bibliography: p. 122.

IN: Sanamed. - ISSN 1452-662X. - Vol. 10, no. 2 (2015), 119-122.

doi: doi:10.5937/sanamed $1502119 \mathrm{M}$

616.24-002-008.87; 616.98:579.887

SM - 50

SEKULIĆ, Stojan, 1954-

Peanut as a Cause of Torsion Meckel's Diverticulum / SekulicStojan, Sekulic-Frkovic Aleksandra, Milankov Andrijana. - Available on: http://scindeks-clanci. ceon.rs/data/pdf/1452-662X/2015/1452-662X1502123S. pdf. - Available on: http://www.sanamed.rs/OJS/index.php/Sanamed/article/view/32. - Abstract; Sažetak. - Bibliography: p. 125.

IN: Sanamed. - ISSN 1452-662X. - Vol. 10, no. 2 (2015), 123-125.

doi: $10.5937 /$ sanamed $1502123 \mathrm{~S}$

616.341-007.64-06; 616.341-007.272-089

SM - 51

SZABÓ, Judit

The Role of IgM-enriched intravenous immunoglobulin in transplantation / Judit Szabó, Anikó Smudla, János Fazakas. - Ill.. - Available on: http:// scindeks-clanci.ceon.rs/data/pdf/1452-662X/2014/1452662X1401063S.pdf. - Available on: http://sanamed. rs/sanamed_pdf/sanamed_9_1/Judit_Szabo.pdf. - Abstract; Sažetak. - Bibliography: p. 69-70.

IN: Sanamed. - ISSN 1452-662X. - Vol. 9, no. 1 (2014), 63-70.

616-089.843-06; 615.37

SM - 52

SKORIC, Jasmina, 1979-

Early Diagnosis of Craniosynostosis in Infants at Primary Health Care / Skoric Jasmina. - Ill.. - Available on: http://scindeks-clanci.ceon.rs/data/pdf/1452662X/2014/1452-662X1403229S.pdf. - Available on: http://sanamed.rs/sanamed_pdf/sanamed_9_3/Skoric Jasmina.pdf. - Abstract; Sažetak. - Bibliography: p. 231.

IN: Sanamed. - ISSN 1452-662X. - Vol. 9, no. 3 (2014), 229-231.

616.714-007.2-053.31

SM - 53

SPASOV, Marko

De Anquin Syndrome-Rare Cause of Low Back Pain : A Case Report with Review of Literature / 
Spasov Marko, Todorov Ilija, Stojanovska-Pemovska Emilija. - Ill. - Available on: http://scindeks-clanci.ceon. rs/data/pdf/1452-662X/2016/1452-662X1602145S.pdf. - Available on: http://www.sanamed.rs/OJS/index. php/Sanamed/issue/view/7. - Notes and bibliographical references with the text. - Abstract; Sažetak. - Bibliography: p. 149.

IN: Sanamed. - ISSN 1452-662X. - Vol. 11, no. 2 (2016), 145-149.

doi: $10.5937 /$ sanamed $1602145 \mathrm{~S}$

616.711-009.7-02; 616.711-007.24-089

\section{SM - 54}

SYSTEMIC non-malignant osteoporosis and reduction of edentulous alveolar / Poštić D. Srđan ... [et al.]. - Ill.. - Available on: http://scindeks-clanci. ceon.rs/data/pdf/1452-662X/2014/1452-662X1401013P. pdf. - Available on: http://sanamed.rs/sanamed_pdf/sanamed_9_1/Srdjan_Postic.pdf. - Other authors: Vujasinović Stupar Nada, Asotić Mithat, Rakočević Zoran. - Abstract; Sažetak. - Bibliography: p. 22-23.

IN: Sanamed. - ISSN 1452-662X. - Vol. 9, no. 1 (2014), 13-23.

616.716.4-007.234

SM - 55

\section{STANKOVIĆ, Svetlana, 1958-}

Acute Complications in Multiple Myeloma Stankovikj Svetlana, Martinova Kata. - Available on: http://www.sanamed.rs/OJS/index.php/Sanamed/article/view/181/97. - Notes and bibliographical references with the text. - Abstract; Sažetak. - Bibliography: p. 119.

IN: Sanamed. - ISSN 1452-662X. - Vol. 12, no. 2 (2017), 115-119.

doi: $10.24125 /$ sanamed.v12i2.181

616.155 .392

SM - 56

SURGICAL Management of Symptomatic Low Back Pain and Monoradicular Leg Pain in Adolescent and Young Adult Patients / Kalevski Svetoslav ... [et al.]. - Photogr., Tables. - Available on: http:// scindeks-clanci.ceon.rs/data/pdf/1452-662X/2014/1452662X1402161K.pdf. - Available on: http://sanamed. rs/sanamed_pdf/sanamed_9_2/Kalevski_Svetoslav.pdf. - Other authors: Haritonov Dimiter, Peev Nikolay, Kalevska Evgenia. - Abstract; Sažetak. - Bibliography: p. 166.

IN: Sanamed. - ISSN 1452-662X. - Vol. 9, no. 2 (2014), 161-166.

616.711-009.7-089-053.6;

616.718-009.7-089-053.6
SM - 57

\section{SCHMITZ, Roland}

Sepsis biomarkers and pathogen detection methods - state of the art / Roland P. H. Schmitz, Frank M. Brunkhorst. - Available on: http://scindeks-clanci.ceon.rs/data/pdf/1452-662X/2014/1452662X1401049S.pdf. - Available on: http://sanamed.rs/ sanamed_pdf/sanamed_9_1/Roland_Schmitz.pdf. - Abstract; Sažetak. - Bibliography: p. 59-61.

IN: Sanamed. - ISSN 1452-662X. - Vol. 9, no. 1 (2014), 49-61.

$$
616.94-074 ; 577.112 .4
$$

\section{SM - 58}

TANDEM Compressin of Medulla Spinalis and CaudaEquina / Zhelyazkov Christo ... [et al.]. - Ill.. Available on: http://www.sanamed.rs/OJS/index.php/ Sanamed/article/view/19/12. - Available on: http://scindeks- clanci.ceon.rs/data/pdf/1452-662X/2015/1452662X1501065Z.pdf. - Abstract; Sažetak. - Bibliography: p. 68.

IN: Sanamed. - ISSN 1452-662X. - Vol. 10, no. 1 (2015), 65-68.

doi: $10.5937 /$ sanamed $1501065 Z$

616.711-007.2-073

SM - 59

\section{TODOROVIĆ, Vladimir, 1964-}

Skin Toxicity of Targeted Therapy: Vemurafenib, First Experiences from Montenegro / Todorovic Vladimir, Martinovic Danilo. - Available on: http:// scindeks-clanci.ceon.rs/data/pdf/1452-662X/2015/1452662X1502109T.pdf. - Available on: http://www.sanamed.rs/OJS/index.php/Sanamed/article/view/32. - Abstract; Sažetak. - Bibliography: p. 113-114.

IN: Sanamed. - ISSN 1452-662X. - Vol. 10, no. 2 (2015), 109-114.

doi: $10.5937 /$ sanamed $1502109 \mathrm{~T}$

616.5-006.81-085.65(497.16)

SM - 60

TREATMENT of Urachal Adenocarcinoma Case Report / Mekic Abazovic Alma ... [et al.]. - Ill., tables. - Available on: http://scindeks-clanci.ceon.rs /data/pdf/1452-662X/2015/1452-662X1502115M.pdf. - Available on: http://www.sanamed.rs/OJS/index. php/Sanamed/article/view/32. - Other authors: Samra Sujelmanovic, Erna Sehic-Kozica, Mirza Mehic, Hakija Beculic, Branislava Jakovljevic. - Abstract; Sažetak. - Bibliography: p. 117.

IN: Sanamed. - ISSN 1452-662X. - Vol. 10, no. 2 (2015), 115-117. 
doi: $10.5937 /$ sanamed $1502115 \mathrm{M}$

616.62-006.6-08

SM - 61

HAJRIĆ, Zlata, 1986-

Outcome of MultivisceralEchinococcosis : A case Report / Hajric Zlata, Pasic Amela, Spahic Razija. - Ill. - Available on: http://www.sanamed.rs/OJS/index.php/Sanamed/article/view/188/101. - Notes and bibliographical references with the text. - Abstract; Sažetak. - Bibliography: p. 113-114.

IN: Sanamed. - ISSN 1452-662X. - Vol. 12, no. 2 (2017), 109-114.

doi: $10.24125 /$ sanamed.v12i2.188

616-002.9-073

SM - 62

\section{HARITONOV, Dimitar, 1968-}

Our Experience with Magerl's Modified Technique for Stabilization of Subaxial Cervical Spine / Haritonov Dimitar, Kalevski Svetoslav, Peev Nikolay. - Ill. - Available on: http://www.sanamed.rs/OJS/index.php/Sanamed/article/view/17/10. - Available on: http://www.sanamed.rs/OJS/index.php/Sanamed/issue/ view/2. - Abstract; Sažetak. - Bibliography: p. 54-55.

IN: Sanamed. - ISSN 1452-662X. - Vol. 10, no. 1 (2015), 51-55.

616.711-001-085; 615.477.3

SM - 63

\section{HADŽIĆ, Devleta}

Caracteristics of Pneumonia Hospitalizations at Pediatric Clinic Tuzla / Hadzic Devleta, Zulic Evlijana. - Ill.. - Available on: http://www.sanamed.rs/ OJS/index.php/Sanamed/article/view/18/11. - Available on: http://www.sanamed.rs/OJS/index.php/Sanamed/issue/view/2. - Abstract; Sažetak. - Bibliography: p. 62-63.

IN: Sanamed. - ISSN 1452-662X. - Vol. 10, no. 1 (2015), 57-63.

doi: $10.5937 /$ sanamed $1501057 \mathrm{H}$

616.24-002-053.2(497.6)

SM - 64

HIV/AIDS Education of Health Care Providers / Ljaljević Agima ... [et al.]. - Ill.. - Available on: http:// scindeks-clanci.ceon.rs/data/pdf/1452-662X/2015/1452662X1501031L.pdf. - Available on: http://www.sanamed.rs/OJS/index.php/Sanamed/issue/view/2. - Abstract; Sažetak. - Bibliography: p. 35.

IN: Sanamed. - ISSN 1452-662X. - Vol. 10, no. 1 (2015), 31-35. doi: $10.5937 /$ sanamed1501031L

616.98:578.828Ć:614.253(497.16)

SM - 65

CATARACT Surgery and Intraocular Lens Power Calculation in a Patient with Anterior Megalophthalmos with Normal Sized Crystalline Lens: Case Report / Glisic Selimir ... [et al.]. - Tables, ill. Available on: http://scindeks-clanci.ceon.rs/data/pdf/ 1452-662X/2015/1452-662X1503209G.pdf. - Available on: http://www.sanamed.rs/OJS/index.php/Sanamed/issue/view/5. - Other authors: Jovanović Miloš, Gaković Aleksandar, Dačić Krnjaja Bojana. - Abstract; Sažetak. - Bibliography: p. 212-213.

IN: Sanamed. - ISSN 1452-662X. - Vol. 10, no. 3 (2015), 209-213.

doi: $10.5937 /$ sanamed $1503209 \mathrm{G}$

616.741-004.1-089; 617.741-77

SM - 66

CLINICAL Relevance of Precore Mutations of Hepatitis B Virus in Chronic Liver Disease / Chaloska Ivanova Viktorija ... [et al.]. - Ill.. - Available on: http://scindeks-clanci.ceon.rs/data/pdf/1452-662X/2014/ 1452-662X1402137C.pdf. - Available on: http://sanamed.rs/sanamed_pdf/sanamed_9_2/Chaloska_Ivanova_Viktorija.pdf. - Other authors: Koloska Valentina, Ganadieva Dimitrova Magdalena, Todorovska Beti, Curakova Elena. - Abstract; Sažetak. - Bibliography: p. 142.

IN: Sanamed. - ISSN 1452-662X. - Vol. 9, no. 2 (2014), 137-142.

616.36-002-06; 616.89-008.454-071

\section{SM - 67}

COMPARATIVE Analysis of Diagnostic Methods in Knee Injuries / Dzoleva-Tolevska Roza ... [et al.]. Tables. - Available on: http://scindeks-clanci.ceon.rs/ data/pdf/1452-662X/2016/1452-662X1601039D.pdf. - Available on: http://www.sanamed.rs/OJS/index. php/Sanamed/issue/view/6. - Abstract; Sažetak. - Bibliography: p. 44-45.

IN: Sanamed. - ISSN 1452-662X. - Vol. 11, no. 1 (2016), 39-45.

doi: $10.5937 /$ sanamed1601039D

616.728.3-07

SM - 68

COMPARATIVE Radiographic Analysis of the Results of Treatment of Hallux Valgus Deformity According to Mitchell and Keller Operative Methods / Georgieva Daniela ... [et al.]. - Tables. Available on: http://scindeks-clanci.ceon.rs/data/pdf/ 1452-662X/2016/1452-662X1601047G.pdf. - Availa- 
ble on: http://www.sanamed.rs/OJS/index.php/Sanamed/issue/view/6. - Abstract; Sažetak. - Bibliography: p. 52.

IN: Sanamed. - ISSN 1452-662X. - Vol. 11, no. 1 (2016), 47-52.

doi: $10.5937 /$ sanamed $1601047 \mathrm{G}$

616.717.7/.9-007.2-073; 616.717.7/.9-089

SM - 69

ČABUKOVSKA Radulovska, Jasminka, 1964-

Lumbosacral Transitional Anatomy Types and Disc Degenerative Changes / Chabukovska Radulovska Jasminka, Matveeva Niki, Poposka Anastasika. Ill.. - Available on: http://scindeks-clanci.ceon.rs/data/pdf/1452-662X/2014/1452-662X1402131C.pdf. - Available on: http://sanamed.rs/sanamed pdf/sanamed _9_2/Chabukovska_Radulovska_Jasminka.pdf. - Abstract; Sažetak. - Bibliography: p. 136.

IN: Sanamed. - ISSN 1452-662X. - Vol. 9, no. 2 (2014), 131-136.

616.711-007-073

616.31 Stomatology

SM - 70

ANĐELIĆ, Ivana, 1989-

The Importance of Oral Health Behaviour of Children for Their Oral Health / Andjelic Ivana, Matijevic Snezana, Andjelic Jasminka. - Available on: http://scindeks-clanci.ceon.rs/data/pdf/1452-662X/2015/ 1452-662X1502101A.pdf. - Available on: http://www. sanamed.rs/OJS/index.php/Sanamed/article/view/32.

- Abstract; Sažetak. - Bibliography: p. 106-107.

IN: Sanamed. - ISSN 1452-662X. - Vol. 10, no. 2 (2015), 101-107.

doi: $10.5937 /$ sanamed1502101A

616.31-053.5(497.16)

SM - 71

DIFFERENTIAL Histomorphometric Changes in Normal and Inflamed Gingival Epithelium / Tanaskovic Stankovic Sanja ... Šet al. ${ }^{1}$. - Ill., tables. Available on: http://scindeks-clanci.ceon.rs/data/pdf/ 1452-662X/2016/1452-662X1603197T.pdf. - Available on: http://www.sanamed.rs/OJS/index.php/Sanamed/issue/view/8. - Other authors: Cabunac Jovan, Kanjevac Tatjana, Milosavljević Zoran. - Notes and bibliographical references with the text. - Abstract; Sažetak. - Bibliography: p. 200-201.

IN: Sanamed. - ISSN 1452-662X. - Vol. 11, no. 3 (2016), 197-201.

doi: $10.5937 /$ sanamed1603197T

616.311.2-002-091.8
SM - 72

KNEŽEVIĆ, Milan, 1948-

Systemic Disorders affecting Dental Pathology / Knezevic R. Milan, Andjelic S. Gordana, Knezevic M. Milena. - Ill.. - Available on: http://scindeks-clanci.ceon. rs/data/pdf/1452-662X/2014/1452-662X1403247K.pdf. - Available on: http://sanamed.rs/sanamed_pdf/sanamed_9_3/Knezevic_Milan.pdf. - Abstract; Sažetak. Bibliography: p. 250-251.

IN: Sanamed. - ISSN 1452-662X. - Vol. 9, no. 3 (2014), 247-251.

616.31-02

SM - 73

MARINOVA-Takorova, Mirela Borislavova, 1978-

Remineralization Potential of a Carbamide Bleaching Agent / Borislavova Marinova-Takorova Mirela, Anastasova Radostina, Panov Vladimir. - Tables. - Available on: http://scindeks-clanci.ceon.rs/data/pdf/1452-662X/2016/1452-662X1601035M.pdf. Available on: http://www.sanamed.rs/OJS/index.php/ Sanamed/issue/view/6. - Abstract; Sažetak. - Bibliography: p. 37-38.

IN: Sanamed. - ISSN 1452-662X. - Vol. 11, no. 1 (2016), 35-38.

doi: $10.5937 /$ sanamed $1601035 \mathrm{~B}$

616.314-008.64-085.31; 615.31:547.495.2

616.8 Neurology. Neuropathology.

Nervous system

SM - 74

\section{ARSOV, Todor}

GLUT-1 Deficiency : from Pathophysilogy and Genetics to Abroad Clinical Spectrum / Arsov Todor. - Available on: http://scindeks-clanci.ceon.rs/data/pdf/1452-662X/2016/1452-662X1602151A.pdf. - Available on: http://www.sanamed.rs/OJS/index.php/Sanamed/issue/view/7. - Notes and bibliographical references with the text. - Abstract; Sažetak. - Bibliography: p. 155.

IN: Sanamed. - ISSN 1452-662X. - Vol. 11, no. 2 (2016), 151-155.

doi: $10.5937 /$ sanamed $1602151 \mathrm{~A}$

$616.831: 575.1$

SM - 75

ENDOVASCULAR Prelude for Delicate Meningeoma Operation: A Case Report / Kostic Aleksandar ... [et al.]. - Ill. - Available on: http://www.sanamed.rs/OJS/index.php/Sanamed/article/view/175/92. - Other authors: RistićSaša, NikolovVesna, Stefanović 
Ivan, DželebdžićZvonimir, Berilažić Luka. - Notes and bibliographical references with the text. - Abstract; Sažetak. - Bibliography: p. 44.

IN: Sanamed. - ISSN 1452-662X. - Vol. 12, no. 1 (2017), 41-44.

doi: $10.24125 /$ sanamed.v1i1.175

616.831-006.328-089

\section{SM - 76}

The INCIDENCE of Nosocomial Infections in Patients with Isolated Severe Traumatic Brain Injury / Valencic Lara ... [et al.]. - Ill.. - Available on: http://scindeks-clanci.ceon.rs/data/pdf/1452-662X/2015/ 1452-662X1503185V.pdf. - Available on: http://www. sanamed.rs/OJS/index.php/Sanamed/issue/view/5. - Abstract; Sažetak. - Bibliography: p. 192.

IN: Sanamed. - ISSN 1452-662X. - Vol. 10, no. 3 (2015), 185-192.

doi: $10.5937 /$ sanamed $1503185 \mathrm{~V}$

616.831-001-06(497.5)"2013/2014";

616-022.1(497.5)"2013/2014"

SM - 77

KALEVSKI, Svetoslav, 1954-

Syndrome of Hydrocephalus in Young and Middle-Aged Adults. Review of the Literatura and Illustrative Cases / Kalevski Svetoslav, Peev Nikolay. - Ill. - Available on: http://scindeks-clanci.ceon.rs/data/pdf/1452-662X/2015/1452-662X1501037K.pdf. Available on: http://www.sanamed.rs/OJS/index.php/ Sanamed/issue/view/2. - Abstract; Sažetak. - Bibliography: p. 44-45.

IN: Sanamed. - ISSN 1452-662X. - Vol. 10, no. 1 (2015), 37-45.

doi: $10.5937 /$ sanamed $1501037 \mathrm{~K}$

616.831

SM - 78

\section{KOLEVSKI, Goran}

Evaluation of Early Ischemic Changes in Stroke Patients Treated with Thrombolytic Therapy / Kolevski Goran, Korneti-Pekevska Kostandina. - Photogr., tables. - Available on: http://scindeks-clanci.ceon. rs/data/pdf/1452-662X/2016/1452-662X1602129K.pdf. - Available on: http://www.sanamed.rs/OJS/index. $\mathrm{php} /$ Sanamed/issue/view/7. - Notes and bibliographical references with the text. - Abstract; Sažetak. - Bibliography: p. 132-133.

IN: Sanamed. - ISSN 1452-662X. - Vol. 11, no. 2 (2016), 129-133.

doi: $10.5937 /$ sanamed $1602129 \mathrm{~K}$

616.831-005.1
SM - 79

MULTIPLE Cerebral Tuberculomas without Focal Neurological Deficit in an Immunocompetent Adult Nigerian : A Case Report / Okokhere O. Peter ... [et al.]. - Ill. - Available on: http://scindeks-clanci. ceon.rs/data/pdf/1452-662X/2016/1452-662X1603221P. pdf. - Available on: http://www.sanamed.rs/OJS/index.php/Sanamed/issue/view/7. - Notes and bibliographical references with the text. - Abstract; Sažetak. - Bibliography: p. 224.

IN: Sanamed. - ISSN 1452-662X. - Vol. 11, no. 3 (2016), 221-224.

doi: $10.5937 /$ sanamed 16032210

616.831-002.5(669)

SM - 80

PEROVIĆ, Zlatana, 1972-

Depression in Patients with Parkinson's Disease with Dementia / Perovic Zlatana, Cukic Mirjana. Tables. - Notes and bibliographical references with the text. - Abstract; Sažetak. - Bibliography: p. 25.

IN: Sanamed. - ISSN 1452-662X. - Vol. 12, no. 1 (2017), 21-25.

doi: $10.24125 /$ sanamed.v1i1.178

616.858:616.89-008.454; 616.858:616.892.3

SM - 81

PETROV, Igor, 1971-

Types of Tremor in Patients with Cerebrovascular Diseases and Cardiovascular Events / Petrov Igor, MulicMersudin, Antonio Georgiev. - Tables. Available on: http://scindeks-clanci.ceon.rs/data/pdf/ 1452-662X/2016/1452-662X1601029P.pdf. - Available on: http://www.sanamed.rs/OJS/index.php/Sanamed/issue/view/6. - Abstract; Sažetak. - Bibliography: p. 33-34.

IN: Sanamed. - ISSN 1452-662X. - Vol. 11, no. 1 (2016), 29-34.

doi: $10.5937 /$ sanamed $1601029 \mathrm{P}$

616.8-009.3-056.24

SM - 82

STOJANOVIĆ, Zlatan, 1977-

Correlation analysis between depressive manifestations and morphological lesion characteristics in patients with stroke / Stojanović Zlatan, Vukadinović Stojanović Sanja. - Ill.. - Available on: http://scindeks-clanci.ceon.rs/data/pdf/1452-662X/2014/1452662X1401031S.pdf. - Available on: http://sanamed.rs/sanamed_pdf/sanamed_9_1/Stojanovic_Zlatan.pdf. - Abstract; Sažetak. - Bibliography: p. 39-40. 
IN: Sanamed. - ISSN 1452-662X. - Vol. 9, no. 1 (2014), 31-40.

616.831-005.6-084; 616.89-008.454-071

\section{SM - 83}

TUMOR surgery within cerebral eloquent areas : a two-institutions experience / Signorelli Francesco...[et al.]. - Available on: http://scindeks-clanci.ceon. rs/data/pdf/1452-662X/2014/1452-662X1402167S.pdf. - Available on: http://sanamed.rs/sanamed_pdf/sanamed_9_2/Signorelli_Francesco.pdf. - Abstract. - Bibliography: p. 174-175.

IN: Sanamed. - ISSN 1452-662X. - Vol. 9, no. 2 (2014), 167-175.

616.831-089(450)"2000/2010";

616.831-089(44)"2000/2010"

\section{SM - 84}

FIRST 5-days Follow-up and Correlation Study Between Urinary CysteinylLeukotrienes and Edema Values in Primary Spontaneous SupratentorialIntracerebral Hemorrhage / Dolnenec-Baneva Natalija ... [et al.]. - Ill.. - Available on: http://scindeks-clanci.ceon.rs/data/pdf/1452-662X/2014/1452662X1402123D.pdf. - Available on: http://sanamed.rs/sanamed_pdf/sanamed_9_2/Dolnenec_Bane-

va_Natalija.pdf. - Other authors: NikodijevikDijana, Petrovska-CvetkovskaDragana, PoposkaAnastasika. Abstract; Sažetak. - Bibliography: p. 129.

IN: Sanamed. - ISSN 1452-662X. - Vol. 9, no. 2 (2014), 123-129.

616.831-005.1-074; 616.89-008.454-071

SM - 85

\section{ŠKRIJELJ, Fadil E., 1958-}

Occipital Lobe Epilepsy or Migraine Headache / Skrijelj E. Fadil, Mulic Mersudin. - Available on: http://scindeks-clanci.ceon.rs/data/pdf/1452-662X/2016/1452-662X1603225S.pdf. Available on: http://www.sanamed.rs/OJS/index.php/ Sanamed/issue/view/7. - Abstract; Sažetak. - Bibliography: p. 228.

IN: Sanamed. - ISSN 1452-662X. - Vol. 11, no. 3 (2016), 225-228.

doi: $10.5937 /$ sanamed $1603225 \mathrm{~S}$

$616.853-079.4 ; 616.857-079.4$

616.89 Psychiatry. Pathological psychiatry. Psychopathology

SM - 86

NUMANOVIĆ, Almedina, 1986-

Correlation Between Domestic Violence against Women and Neuroticism / Numanovic S. Alme- dina, Jovicic M. Milena. - Ill.. - Načindostupa (URL): http://scindeks-clanci.ceon.rs/data/pdf/1452-662X/2014/ 1452-662X1403223N.pdf. - Abstract; Sažetak. - Bibliography: p. 228.

IN: Sanamed. - ISSN 1452-662X. - Vol. 9, no. 3 (2014), 223-228.

$616.891-055.2 ; 316.624: 316.356 .2$

SM - 87

\section{RASPOPOVIC, Milena, 1986-}

The Connection between Perfectionism and Anxiety in University Students / Raspopovic Milena. Tables. - Available on: http://scindeks-clanci.ceon. rs/data/pdf/1452-662X/2015/1452-662X1503199R.pdf. - Available on: http://www.sanamed.rs/OJS/index. php/Sanamed/issue/view/5. - Abstract; Sažetak. - Bibliography: p. 203.

IN: Sanamed. - ISSN 1452-662X. - Vol. 10, no. 3 (2015), 199-203.

doi: $10.5937 /$ sanamed $1503199 \mathrm{R}$

616.89-008.441-057.874

\section{Surgery}

SM - 88

INTRA-Abdominal Infection and Acute Abdomen-Epidemiology, Diagnosis / Jovanovic Dusan ... [et al.]. - Ill., tables. - Available on: http://www.sanamed.rs/OJS/index.php/Sanamed/article/view/20.

Available on: http://www.sanamed.rs/OJS/index.php/ Sanamed/issue/view/2. - Abstract; Sažetak. - Bibliography: p. 77-78.

IN: Sanamed. - ISSN 1452-662X. - Vol. 10, no. 1 (2015), 69-78.

doi: $10.5937 /$ sanamed $1501069 \mathrm{~J}$

617.55-002; 616.381-002-08

SM - 89

\section{RADIVOJEVIĆ, Uroš}

Giant Hand Lipoma : Case Report of a Rare Localization of a Common Type of Tumor / Radivojević Uroš, Ilić B. Milena, Vulović D. Dejan. - Ill. Available on: http://scindeks-clanci.ceon.rs/data/pdf/ 1452-662X/2016/1452-662X1602141R.pdf. - Available on: http://www.sanamed.rs/OJS/index.php/Sana$\mathrm{med} /$ issue/view/7. - Notes and bibliographical references with the text. - Abstract; Sažetak. - Bibliography: p. 144.

IN: Sanamed. - ISSN 1452-662X. - Vol. 11, no. 2 (2016), 141-144.

doi: $10.5937 /$ sanamed $1602141 \mathrm{R}$

617.576-006.326

617.7 Ophthalmology 
SM - 90

\section{KARGANOV, Mikhail}

Laser Correlation Spectroscopy (LCS) and its Clinical Perspectives in Ophthalmology / Karganov Mikhail, Eskina Erika, Stepanova Maria. - Ill.. - Available on: http://scindeks-clanci.ceon.rs/data/pdf/1452662X/2015/1452-662X1503229K.pdf. - Available on: http://www.sanamed.rs/OJS/index.php/Sanamed/issue/view/5. - Abstract. - Bibliography: p. 232-233.

IN: Sanamed. - ISSN 1452-662X. - Vol. 10, no. 3 (2015), 229-233.

doi: $10.5937 /$ sanamed $1503229 \mathrm{~K}$

617.741-089

SM - 91

MISTAKES in the Diagnosis and Treatment of Primary Angle-Closure Glaucoma : Case Report / MarićVesna ... [et al.]. - Ill. - Available on: http://scindeks-clanci.ceon.rs/data/pdf/1452-662X/2016/1452662X1602135M.pdf. - Available on: http://www.sanamed.rs/OJS/index.php/Sanamed/issue/view/7. - Other authors: Marković Vijica, Božić Marija, Marijanović Ivan. - Notes and bibliographical references with the text. - Abstract; Sažetak. - Bibliography: p. 140.

IN: Sanamed. - ISSN 1452-662X. - Vol. 11, no. 2 (2016), 135-140.

doi: $10.5937 /$ sanamed $1602135 \mathrm{M}$

617.7-007.681-085.06

\section{SM - 92}

OCULAR Hypertension Risk Factors and Therapy? / Janicijevic Katarina ... [et al.]. - Tabela. - Available on: http://scindeks-clanci.ceon.rs/data/pdf/1452662X/2015/1452-662X1503193J.pdf. - Available on: http://www.sanamed.rs/OJS/index.php/Sanamed/issue/view/5. - Other authors: Kocić Sanja, Todorović Dušan, Šarenac Vulović Tatjana. - Abstract; Sažetak. Bibliography: p. 197-198.

IN: Sanamed. - ISSN 1452-662X. - Vol. 10, no. 3 (2015), 193-198.

doi: $10.5937 /$ sanamed $1503193 \mathrm{~J}$

617.7:616.12-008.331.1"2009/2015"

SM - 93

PARASITIC Eye Infection by AscarisLumbricoides - Case Report / Janićijević-Petrović A. Mirjana ... [et al.]. - Photogr. - Available on: http://scindeks-clanci.ceon.rs/data/pdf/1452-662X/2014/1452662X1402181J.pdf. - Available on: http://sanamed.rs/sanamed_pdf/sanamed_9_2/Janicijevic_Petrovic.pdf. - Other authors: Šarenac-Vulović Tatjana, Vulović Dejan, Janićijević Katarina, Popović Andrijana. Abstract; Sažetak. - Bibliography: p. 184.
IN: Sanamed. - ISSN 1452-662X. - Vol. 9, no. 2 (2014), 181-184.

617.7-022:595.132; 616.995.132

SM - 94

PREVENTION of Adenoviral Eye Infection Review / Janicijevic Katarina ... [et al.]. - Available on: http://www.sanamed.rs/OJS/index.php/Sanamed/article/view/161/86. - Other authors: Kocić Sanja, Radovanović Snežana, Radević Svetlana, Vasiljević Dragan, Đonović Nela, Šarenac Tatjana. - Notes and bibliographical references with the text. - Abstract; Sažetak. - Bibliography: p. 55-56.

IN: Sanamed. - ISSN 1452-662X. - Vol. 12, no. 1 (2017), 51-56.

doi: $10.24125 /$ sanamed.v1i1.161

617.711-002-084; 616.98:578.826

SM - 95

CURRENT Concepts in Therapy of Uveal Melanoma / Detanac A. Dzenana ... [et al.]. - Available on: http://scindeks-clanci.ceon.rs/data/pdf/1452-662X/2015/ 1452-662X1502137D.pdf. - Available on: http://www. sanamed.rs/OJS/index.php/ Sanamed/article/view/32. - Other authors: Jančić Snežana, Rakočević Milena, Ćeranić Merima. - Abstract; Sažetak. - Bibliography: p. 141.

IN: Sanamed. - ISSN 1452-662X. - Vol. 10, no. 2 (2015), 137-141.

doi: $10.5937 /$ sanamed1502137D

617.72-006.81-08

SM - 96

ŠARENAC-Vulović, Tatjana, 1973-

Primary Open-Angle Glaucoma and Farmacoeconomics - Review / Sarenac Vulovic Tatjana, Janicijevic Katarina. - Available on: http://scindeks-clanci.ceon. rs/data/pdf/1452-662X/2016/1452-662X1603243S.pdf. - Available on: http://www.sanamed.rs/OJS/index. $\mathrm{php} /$ Sanamed/issue/view/7. - Notes and bibliographical references with the text. - Abstract; Sažetak. - Bibliography: p. 247-248.

IN: Sanamed. - ISSN 1452-662X. - Vol. 11, no. 3 (2016), 243-248.

doi: $10.5937 /$ sanamed $1603243 \mathrm{~S}$

617.7-007.681-085; 657.474:616-08

\section{Gynaecology. Obstetrics}

SM - 97

The BURDEN of Vesico-Vaginal Fistula in Ile-Ife, South Western Nigeria / Fehintola O. Akintunde ... [et al.]. - Tables, ill. - Available on: http:// www.sanamed.rs/OJS/index.php/Sanamed/article/vi- 
ew/182/98. - Notes and bibliographical references with the text. - Abstract; Sažetak. - Bibliography: p. 85.

IN: Sanamed. - ISSN 1452-662X. - Vol. 12, no. 2 (2017), 79-85.

doi: 10.24125/sanamed.v12i2.182

618.6-007.253(669)"1984/2013"

SM - 98

ECTOPIC Choriocarcinoma in a Preteen in Ogbomoso, South-West Nigeria. A Case Report / Ogunlaja A. Olumuyiwa ... [et al.]. - Ill. - Available on: http://scindeks-clanci.ceon.rs/data/pdf/1452-662X/2016/ 1452-662X1603217O.pdf. - Available on: http:/www. sanamed.rs/OJS/index.php/Sanamed/issue/view/7.

Notes and bibliographical references with the text. Abstract; Sažetak. - Bibliography: p. 220.

IN: Sanamed. - ISSN 1452-662X. - Vol. 11, no. 3 (2016), 217-220.

doi: $10.5937 /$ sanamed 16032170

618.11-006.6-053.5(669)

\section{SM - 99}

REPRODUCTIVE Outcome, Duration of Pregnancy and Mode of Delivery After Hysteroscopic Metroplasty in Patients with Infertility / Tofoski Gligor ... [et al.]. - Ill. - Available on: http://scindeks-clanci.ceon.rs/data/pdf/1452-662X/2016/1452662X1602117T.pdf. - Available on: http://www.sanamed.rs/OJS/index.php/Sanamed/issue/view/7. - Other authors: Dimitrov Goran, Hadži Lega Marija, Džikova Elena. - Notes and bibliographical references with the text. - Abstract; Sažetak. - Bibliography: p. 121-122.

IN: Sanamed. - ISSN 1452-662X. - Vol. 11, no. 2 (2016), 117-122.

doi: $10.5937 /$ sanamed $1602117 \mathrm{~T}$

618.14-007.2-089.844; 618.3

\author{
Correspondence to / Autor za korespondenciju \\ Jašović Ivana \\ National library of Serbia, Belgrade \\ Serbia \\ email: ivana.jasovic@nb.rs
}

SM - 100

FUNCTIONAL Complications Following Breast Cancer Therapy and the Role of Rehabilitation in Recovery of Functional Status - A Case Report / Popović-Petrović Svetlana ... [et al.]. - Ill. - Other authors: Kovač Aleksandra, Novakov Ivana, Tatić Milanka. - Notes and bibliographical references with the text. - Abstract; Sažetak. - Bibliography: p. 40.

IN: Sanamed. - ISSN 1452-662X. - Vol. 12, no. 1 (2017), 37-40.

doi: $10.24125 /$ sanamed.v1i1.172

618.19-006.6-089.168; 615.8

SM - 101

HADŽI-Lega, Marija, 1973-

Cervical Length and Phosphorilated Insulin like Growth Factor Binding Protein-1 as the Predictors of Spontaneus Preterm Delivery in Symptomatic Women / Hadži-Lega Marija, Daneva Markova Ana, Stefanovic Milan. - Ill.. - Available on: http://scindeksclanci.ceon.rs/data/pdf/1452-662X/2014/1452-662X1 402143H.pdf. - Available on: http://sanamed.rs/sanamed_pdf/sanamed_9_2/Hadzi_Lega_Marija.pdf.

Abstract; Sažetak. - Bibliography: p. 149-150.

IN: Sanamed. - ISSN 1452-662X. - Vol. 9, no. 2 (2014), 143-150.

618.39-021.3-07:618.146

\section{DECLARATION OF INTEREST}

The autors declare that there are no conflicts of interests.

\section{Licensing}

This work is licensed under a Creative Commons Attribution 4.0 International (CC BY 4.0) License 